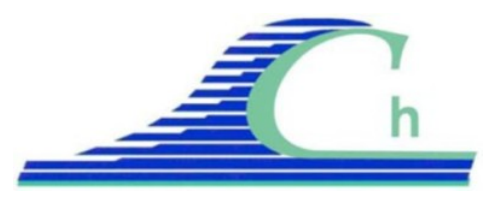

XII ${ }^{\text {èmes }}$ Journées Nationales Génie Côtier - Génie Civil

Cherbourg, 12-14 juin 2012

DOI:10.5150/jngcgc.2012.008-G（C) Editions Paralia CFL

disponible en ligne - http://www.paralia.fr - available online

\title{
Origine des courants d'arrachement bien établis
}

\author{
Roland GARNIER ${ }^{1}$, Albert FALQUÉS ${ }^{2}$, Nicholas DODD ${ }^{3}$
}

1. Instituto de Hidráulica Ambiental, Universidad de Cantabria, PCTCAN, C/ Isabel Torres 15, 39011 Santander, Espagne.

garnierr@unican.es

2. Departament de Física Aplicada, Universitat Politècnica de Catalunya, C/ Jordi

Girona 1-3, 08034 Barcelona, Espagne.

falques@fa.upc.edu

3. Department of Civil Engineering, Faculty of Engineering, University of Nottingham, NG72RD Nottingham, England, Royaume-Uni.

nicholas.dodd@nottingham.ac.uk

\section{Résumé :}

Les courants d'arrachement (rip currents) sont des courants particulièrement intenses, induits par les vagues et orientés vers le large. Sur la plupart des plages sableuses ouvertes, ils apparaissent de manière quasi-périodique le long de la côte et, lorsqu'ils sont bien établis (maintenus $\sim 1$ heure), sont associés à des irrégularités topographiques (les systèmes de barres/baïnes, ou les systèmes de barres en croissant par exemple). Comprendre l'origine de ces courants est un enjeu scientifique majeur depuis plusieurs décennies. Les différents mécanismes évoqués pour expliquer leur formation peuvent être classés selon deux théories: (1) la théorie selon laquelle les courants d'arrachement pourraient apparaître sur des plages rectilignes (ondes de bords, instabilités hydrodynamiques), dans ce cas, la morphologie tridimensionnelle associée serait forcée par l'hydrodynamique (mécanismes forcés); et (2) la théorie selon laquelle l'évolution $\mathrm{du}$ fond est nécessaire pour former ces courants (mécanismes d'auto-organisation morphodynamique). Nous présenterons les dernières études de modélisation hydro et morphodynamique. Elles montrent que, pour des conditions naturelles, la théorie d'auto-organisation morphodynamique est la plus convaincante. Nous évoquerons également des situations quasi-forcées et montrerons qu'elles peuvent s'apparenter à cette théorie.

\section{Mots-clés :}

Courants d'arrachement - Rips - Instabilités hydrodynamiques - Auto-organisation morphodynamique

\section{Introduction}

Les courants d'arrachement (rip currents), localisés dans la zone de surf, sont des courants particulièrement intenses induits par les vagues et orientés vers le large, pouvant atteindre des vitesses de 1-2 m/s. Le fort intérêt qu'ils suscitent parmi le grand 
public et les décideurs politiques est lié à la sécurité des plages (risque de noyades), aux problèmes d'érosion des plages (transport sédimentaire associé) et au transport de polluants.

Sur la plupart des plages sableuses ouvertes (telles que les plages d'Aquitaine), ils apparaissent de manière quasi-périodique le long de la côte. Lorsqu'ils sont bien établis (maintenus durant un temps caractéristique de l'ordre de 1 heure ou plus) ils sont généralement associés à des irrégularités bathymétriques donnant lieu à une intrigante et persistante forme quasi-rythmique aux fonds sableux (SHORT, 1999 et VAN ENCKEVORT et al., 2004, par exemple). Dans la zone de surf, ces structures sont référenciées comme systèmes de barres rythmiques. En particulier, ces structures peuvent être observées sur la côte atlantique française sous la forme de systèmes de barres/baïnes, ou de systèmes de barres en croissant (LAFON et al., 2002 et CASTELLE et al., 2007).

Comprendre l'origine de ces courants est un enjeu scientifique majeur depuis plusieurs décennies. Il existe de nombreuses révisions scientifiques répertoriant les centaines d'études consacrées aux courants d'arrachement, citons par exemple les deux dernières révisions, publiées par MACMAHAN et al. (2006) et DALRIMPLE et al. (2011). Ces études rapportent les dernières avancées permettant de comprendre la dynamique des courants d'arrachement d'après les résultats de modèles mathématiques, d'expériences en laboratoire et d'observations de plages réelles. Afin de considérer tous les éléments permettant de comprendre la dynamique des courants d'arrachement, il est important $\mathrm{d}$ 'inclure les études de morphologie de plages, et, en particulier les études de dynamique des barres sableuses (BLONDEAUX, 2001, DODD et al., 2003, COCO \& MURRAY, 2007 et FALQUES et al., 2008).

L'objectif principal de cette étude est de recenser et de classer les principales possibilités de formations des courants d'arrachement établis évoquées dans les études antérieures afin de dégager les hypothèses de formation les plus convaincantes. Cette étude est restreinte au cas des plages ouvertes.

\section{Les différents types de théorie}

L'étude expérimentale de BOWEN \& INMAN (1969) a montré qu'il était possible de générer les courants d'arrachement sur fond uniforme et non érodables, grâce à un forçage particulier et dans des conditions de laboratoire. Sur des plages réelles, il est difficile de prouver la présence de courant d'arrachement sans structure topographique associée (MACMAHAN et al., 2005). Cependant, DALRIMPLE et al. (2011) évoquent l'existence de courants d'arrachement à caractère épisodique (persistant durant moins de 15 minutes) qui pourraient être indépendants de la morphologie de la plage. Ce sujet reste ouvert et ne sera pas abordé dans cette étude qui, nous le rappelons, se centrera sur les courants d'arrachement biens établis, et donc liés à la structure morphologique. 


\section{XII ${ }^{\text {èmes }}$ Journées Nationales Génie Côtier - Génie Civil \\ Cherbourg, 12-14 juin 2012}

Les différents mécanismes évoqués pour expliquer la formation des barres rythmiques et courants associés sont classés communément selon deux théories (figure 1): (1) le forçage hydrodynamique, théorie selon laquelle les structures morphologiques seraient le résultat d'une réponse passive à un forçage hydrodynamique et (2) l'autoorganisation morphodynamique, théorie selon laquelle les structures morphologiques émergeraient à partir d'instabilités internes du système morphodynamique par un processus d'auto-organisation couplant l'évolution du fond et l'hydrodynamique.

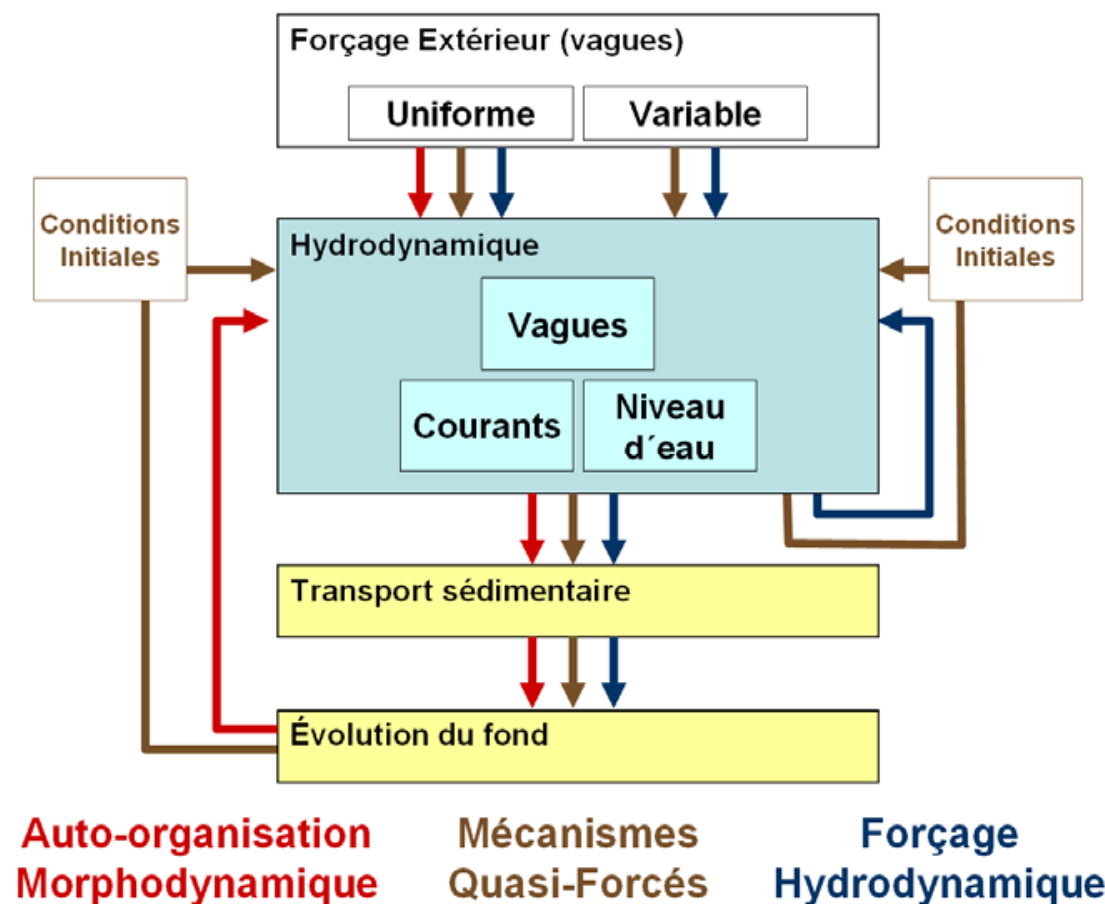

Figure 1. Principe de la formation des courants d'arrachement et morphologie associée par des mécanismes d'auto-organisation hydrodynamique ou morphodynamique.

En d'autres termes, selon la première théorie (forçage hydrodynamique), les courants d'arrachement pourraient apparaître sur des plages rectilignes (uniformes dans la direction longitudinale). Dans cette étude, nous évoquerons les différents types de mécanismes pouvant expliquer la formation des courants d'arrachement par forçage hydrodynamique et nous verrons qu'ils peuvent être dus à des instabilités du système hydrodynamique et à des processus d'auto-organisation purement hydrodynamique.

Selon la deuxième théorie (auto-organisation morphodynamique) l'évolution du fond est nécessaire pour former ces courants et la réponse du système hydromorphodynamique est souvent complexe et imprévisible. Cependant en supposant certaines simplifications sur le système dynamique, nous verrons qu'il est possible de comprendre la formation des systèmes de barres/chenaux. 
Enfin, nous définirons les mécanismes quasi-forcés (figure 1) comme les mécanismes d'auto-organisation morphodynamique influencés par un forçage non-uniforme de l'hydrodynamique ou par la non-uniformité des conditions initiales (bathymétrie initiale).

\section{Forçage hydrodynamique}

Si les systèmes de barres rythmiques se formaient dû à un forçage hydrodynamique, les courants d'arrachement pourraient apparaître, théoriquement, sur des plages rectilignes. Les mécanismes qui peuvent expliquer la génération des courants d'arrachement sur fond uniforme peuvent être liés à des instabilités hydrodynamiques. Il s'agit (1) de la théorie des ondes de bords (BOWEN \& INMAN, 1969) générées par des interactions de vagues qui peuvent être le résultat d'une instabilité et (2) des théories d'instabilités hydrodynamiques dues à des interactions au sein du système hydrodynamique en général (FALQUÉS et al., 1999), comme par exemple les interactions vagues - courants - niveau d'eau moyen.

\subsection{Ondes de bord}

Les ondes de bord sont des vagues infra-gravitaires piégées près de la côte dû à la réfraction. Elles ont une structure périodique le long de la côte et se propagent généralement parallèlement à la côte. Cependant, pour certaines configurations, les études théoriques et expérimentales de laboratoire montrent que ces ondes peuvent être stationnaires, et, dans ce cas, qu'elles peuvent être le moteur entraînant la formation des courants d'arrachement.

Cette théorie, introduite par BOWEN \& INMAN (1969), a été adoptée par la majorité de la communauté scientifique dans les années 70 , et a été particulièrement reconnue pour expliquer la formation des croissants de plage (beach cusps, GUZA \& INMAN, 1975). Il est vrai que cette théorie à été très bien validée en laboratoire et qu'elle permet de justifier approximativement la longueur d'onde des systèmes morphologiques observés sur les plages réelles.

Cependant, la critique publiée par SONU (1971) ainsi que de nombreuses études plus récentes montrent que cette hypothèse ne peut pas expliquer tous les cas observés, notamment sur les plages ouvertes. En particulier, l'amplitude des ondes de bord est faible et est atténuée par la friction des fonds sableux : aucune étude n'a pu prouver l'existence des ondes de bords sur des plages ouvertes. En se centrant sur le cas des plages d'Aquitaine (CASTELLE et al., 2007), la variabilité des longueurs d'ondes observées (600-800 m pour les barres en croissant externes) ne peut pas être expliquée par la théorie des ondes de bord. De plus, même si BOWEN \& INMAN (1969) montrent qu'il est possible d'expliquer la formation d'un double système de barres en croissant (les systèmes internes et externes), leur approche prévoit la même longueur d'onde pour les deux systèmes, alors que la longueur d'onde du système interne est, en 


\section{XII ${ }^{\text {èmes }}$ Journées Nationales Génie Côtier - Génie Civil \\ Cherbourg, 12-14 juin 2012}

réalité, généralement inférieure (300-500 m) à la longueur d'onde du système externe. Enfin, selon leur théorie les plages à fort marnage ne sont pas propices à l'apparition des barres en croissant.

Ainsi, même si pour des conditions spécifiques (expériences en laboratoire, ou pour certaines configurations de plages en baies) les ondes de bords peuvent jouer un rôle dans la formation des courants d'arrachement, cette théorie n'explique pas toutes les situations observées. De plus, dans aucun cas, on a pu prouver qu'en l'absence d'onde de bord, les courants d'arrachement ne pouvaient apparaître.

3.2 Instabilités hydrodynamiques (interactions vagues - courants - niveau d'eau moyen) A la différence des mécanismes évoqués précédemment (théories des ondes de bord stationnaires), ces instabilités hydrodynamiques peuvent expliquer la formation des courants d'arrachement sans conditions particulières aux frontières latérales du domaine (réflexion contre les parois latérales d'un bassin favorisant l'apparition des ondes de bords stationnaires, GUZA \& INMAN, 1975) et sans aucune variabilité dans le forçage des vagues.

Les études théoriques de HINO (1974) et de DALRYMPLE \& LOZANO (1978) ont fait l'hypothèse qu'une instabilité hydrodynamique pouvaient générer les courants d'arrachement, cependant aucune étude n'a rigoureusement vérifié cette hypothèse avant l'étude de stabilité linéaire de FALQUÉS et al. (1999), puis celle de YU (2006). Il ressort de ces études que les interactions vagues-courant-niveau d'eau peuvent générer une instabilité hydrodynamique. Il faut noter, cependant, que ces études considèrent que la zone de surf est saturée (i.e. en zone de surf, la hauteur de vague moyenne est proportionnelle à la profondeur d'eau moyenne), et que l'instabilité est générée principalement dans la zone de levée (shoaling zone).

La figure 2 schématise un exemple d'instabilité hydrodynamique. Considérons une perturbation du niveau d'eau moyen et la circulation associée (figure $2 \mathrm{a}$, les zones claires (sombres) indiquent une élévation (baisse) du niveau d'eau), générées par une perturbation de la hauteur de vague et transmise par les tenseurs de radiation. La perturbation du niveau d'eau et les courants vont augmenter la courbure des fronts de vagues dus à la réfraction et aux interactions vagues-courants (figure $2 \mathrm{~b}$ ). Ces fronts d'onde, à leur tour (rétroaction positive), vont générer une amplification de la perturbation du niveau, et de plus forts courants (figure 2c). 


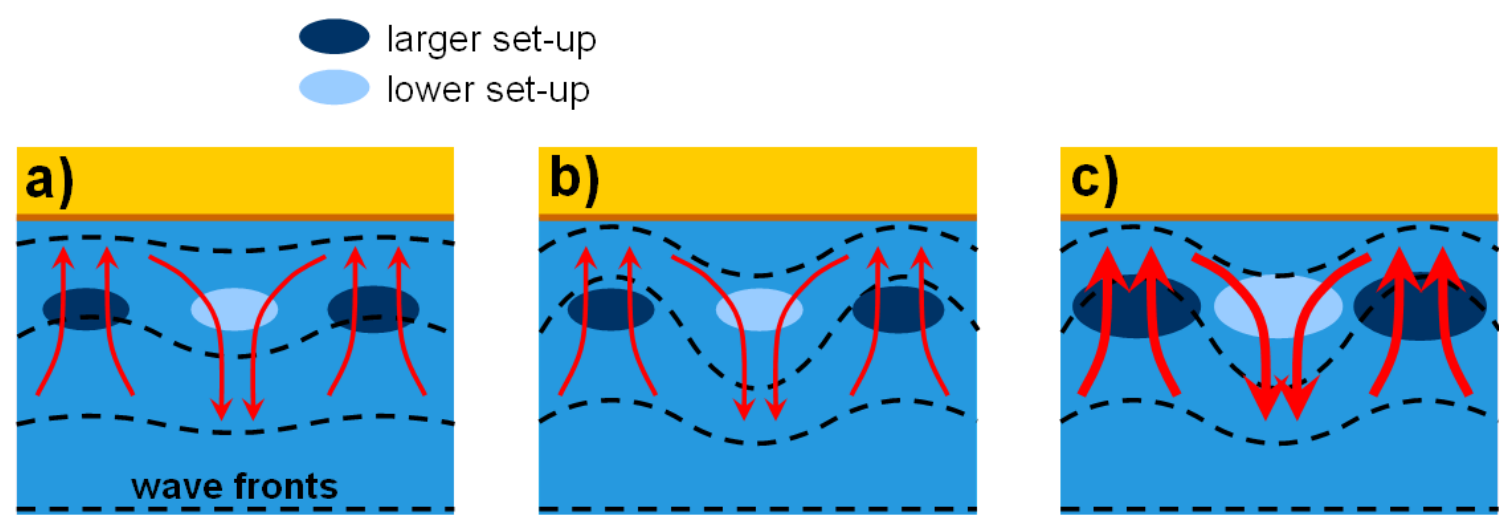

Figure 2. Exemple d'un mécanisme d'instabilité hydrodynamique due aux interactions vagues/courant. (a) Perturbation du niveau d'eau moyen et circulation associée. (b) Amplification de la déformation des fronts de vagues due aux courants. (c) Rétroaction positive: amplification de la circulation et de la perturbation du niveau d'eau.

La dernière étude d'instabilité hydrodynamique a été réalisée par HASAN et al. (2009), et est la première étude à considérer des vagues irrégulières. Comme dans les études précédentes, le modèle numérique utilisé est un modèle de type $2 \mathrm{DH}$ (équations moyennées sur une colonne d'eau et sur une période de vague). Le modèle, MORFO60, utilise l'analyse de stabilité linéaire et est utilisé en mode hydrodynamique (MORFO60, CALVETE et al., 2005). L'étude montre que, pour des conditions de vagues irrégulières (déferlement de type THORNTON \& GUZA, 1983), 1'effet de la perturbation du niveau d'eau sur le déferlement entraine une rétroaction négative empêchant les instabilités de se développer. Cette étude remet donc en question la théorie de formation de courants d'arrachement comme instabilité hydrodynamique.

\section{Mécanismes d'auto-organisation morphodynamiques}

Le premier à avoir formulé l'hypothèse d'auto-organisation morphodynamique est SONU (1968). Il explique comment les structures rythmiques peuvent être dues à des mécanismes d'interaction entre l'hydrodynamique et la morphologie. La démonstration à été présentée par BARCILON \& LAU (1973) et par HINO (1974) au moyen d'analyse de stabilité linéaire. Même si HINO (1974) interprète la formation des courants $\mathrm{d}$ 'arrachement comme une instabilité hydrodynamique, son modèle intègre le couplage avec l'évolution du fond et, comme FALQUÉS et al. (1999) le souligne, ce couplage est probablement nécessaire pour générer l'instabilité.

Différents types de mécanismes peuvent expliquer la formation de courants d'arrachement par auto-organisation morphodynamique (FALQUÉS et al., 2000). Pour des conditions de vagues stationnaires et uniformes et pour une plage initialement rectiligne soumise à l'unique forçage de la houle, la formation des courants d'arrachement et de la morphologie associée dépendra essentiellement de la direction 


\section{XII ${ }^{\text {èmes }}$ Journées Nationales Génie Côtier - Génie Civil \\ Cherbourg, 12-14 juin 2012}

des vagues incidentes, du profil bathymétrique et du transport sédimentaire (ce dernier dépend des caractéristiques du sédiment, de la profondeur d'eau, mais aussi de l'hydrodynamique). Compte-tenu des incertitudes concernant les formulations du transport sédimentaire, les résultats des modèles numériques pouvant expliquer la génération des courant $\mathrm{d}$ 'arrachement dépendront du choix de la formule de transport utilisée. Il est donc important de pouvoir classer les formules de transport existantes selon le type d'instabilité qu'elles peuvent générer comme l'on fait les études de FALQUÉS et al. (2000), CABALLERIA et al. (2002), RIBAS et al. (2003) et GARNIER et al. (2006).
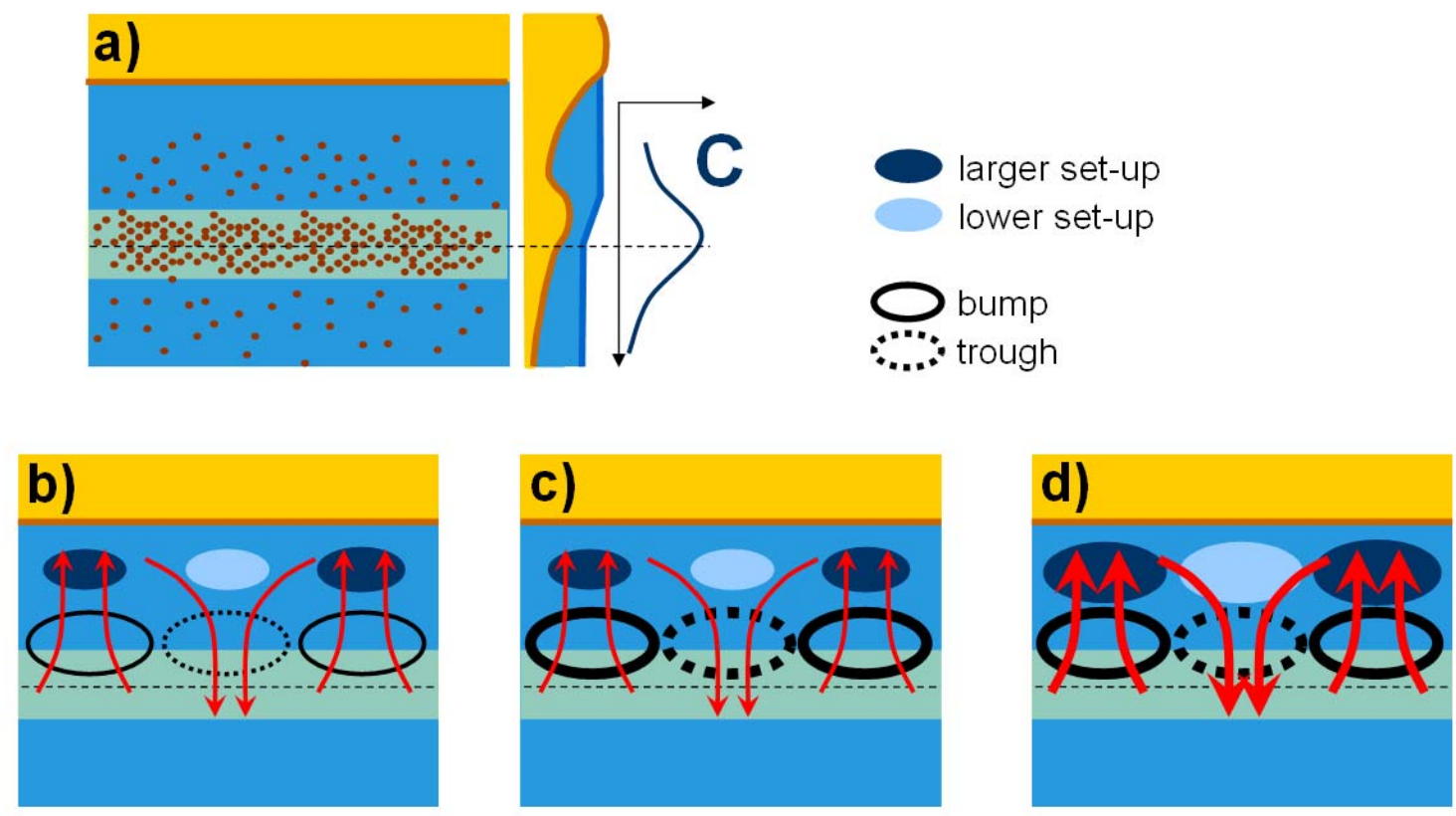

Figure 3. Mécanisme d'instabilité morphodynamique dans le cas de vagues frontales.

(a) État de base (uniforme) et concentration des sédiments moyennée sur la profondeur d'eau. Concentration maximale au niveau de la barre parallèle. (b) Perturbation de la topographie et circulation associée. (c) Amplification de la perturbation de la topographie due au courant. (d) Rétroaction positive: intensification des courants.

La figure 3 schématise le mécanisme d'auto-organisation morphodynamique pour des vagues frontales dans le cas d'une plage à barre parallèle (marquée par la zone claire, figure 3a). Nous supposons que les conditions initiales sont uniformes dans la direction longitudinale, aussi bien les vagues incidentes que la bathymétrie. De plus, sans aucune perturbation du fond, nous supposons que, sous l'action de la houle, le fond reste immobile (équilibre cross-shore) et que toutes les variables hydrodynamiques sont en équilibre et uniformes longitudinalement. Dans ce cas, le profil de concentration de sédiment moyenné sur la profondeur d'eau (C) présente un maximum au niveau de la barre parallèle (figure 3a). Si on perturbe la topographie (en imposant par exemple une 
petite perturbation aléatoire) le fond et l'hydrodynamique peuvent s'auto-organiser. Le mécanisme d'instabilité, c'est à dire la rétroaction positive s'explique de la manière suivante. En considérant une succession de barres/chenaux dans la zone de surf, des circulations se créent, caractérisées par un courant vers le large dans les chenaux (figure $3 b$ ). Si ces structures morphologiques se trouvent dans une zone où la concentration augmente vers le large (i.e. sur la partie interne de la barre parallèle), le transport sédimentaire va amplifier ces structures (les chenaux vont s'éroder et les barres vont se développer, figure 3c). A leur tour, ces structures plus développées vont amplifier la circulation associée, la rétroaction est positive (figure $3 \mathrm{~d}$ ).

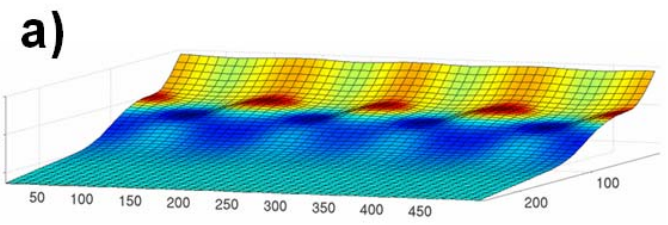

b)

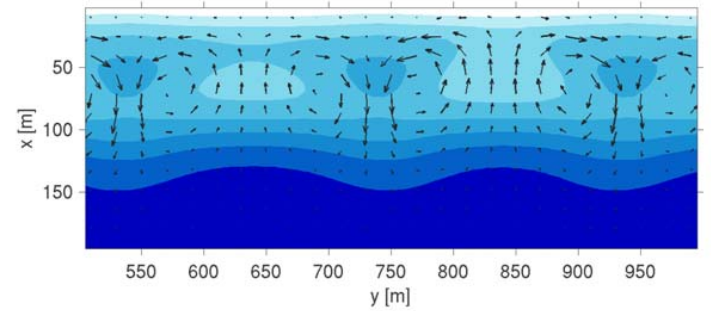

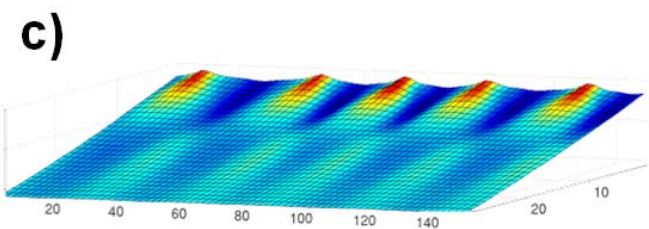

d)

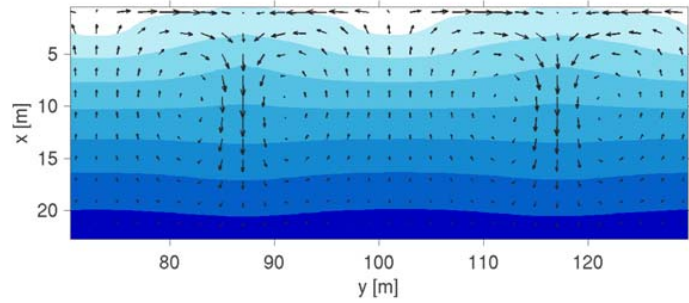

Figure 4. Résultats d'auto-organisation morphodynamique obtenus avec le modèle MORFO55. États finaux. (a, b) Barres en croissant développées au niveau de la barre parallèle (plage à barre parallèle). (c, d) Barres transverses attachées au trait de côte

(plage plane). ( $a, c)$ Bathymétrie 3D. $(b, d)$ Contours bathymétriques et courants associés.

Le modèle 2DH non-linéaire MORFO55 (CABALLERIA et al., 2002 et GARNIER et al., 2006) a été appliqué pour illustrer le mécanisme décrit précédemment: la figure 4a,b montre le développement d'une structure rythmique de barres en croissant au niveau de la barre parallèle, avec une longueur d'onde de $200 \mathrm{~m}$ (résultats commentés par GARNIER et al., 2008). Ce mécanisme peut aussi s'appliquer pour les plages planes (dépourvues de barres parallèles) comme le montre la figure 4c,d. Dans ce cas, le profil de concentration présente un maximum près du bord, et des barres transverses (attachées au trait de côte) peuvent se générer avec une longueur d'onde de 30m (voir GARNIER et al., 2006).

Alors que les premières études étaient centrées sur la formation initiale des systèmes de barres rythmiques et courants associés en utilisant la théorie de stabilité linéaire (HINO 


\section{XII ${ }^{\text {èmes }}$ Journées Nationales Génie Côtier - Génie Civil \\ Cherbourg, 12-14 juin 2012}

1974, CHRISTENSEN et al., 1994 et CALVETE et al., 2005, par exemple), toutes les dernières études sont consacrées à l'évolution non-linéaire et au comportement à long terme (GARNIER et al., 2008, 2008b, 2010 et CASTELLE \& RUESSINK, 2011 et THIEBOT et al., 2012). En particulier, les interactions non-linéaires telles que l'appariement et la séparation de certaines barres peuvent expliquer la variabilité des longueurs d'onde observée dans la nature.

\section{Mécanismes quasi-forcés}

Comme nous l'avons défini antérieurement, nous appelons les mécanismes quasi-forcés, les mécanismes d'auto-organisation morphodynamique influencés par un forçage nonuniforme de l'hydrodynamique ou par la non-uniformité des conditions initiales (bathymétrie initiale). Ils se distinguent des mécanismes de forçage hydrodynamique car ils incluent les cas où (1) sans rétroaction des fonds sableux, les courants d'arrachement ne pourraient pas apparaître (2) en supposant des conditions initiales uniformes, des courants d'arrachement pourraient apparaître selon les mécanismes d'auto-organisation morphodynamique décrits précédemment.

Dans ce cas, les courants d'arrachement et les structures morphologiques associées devraient se former à la même distance de la côte que le prévoit la théorie d'autoorganisation morphodynamique. En particulier, pour des vagues frontales, l'intensité maximale des courants devrait être mesurée dans des zones instables, c'est à dire dans les zones à forts gradients de concentration (figure 3 ).

Cependant, malgré cette similitude avec les mécanismes purement auto-organisés, les mécanismes quasi-forcés peuvent générer de grandes différences dans la dynamique des systèmes rythmiques (augmentation de la variabilité des longueurs d'ondes, accélération des processus de formation, différence de longueur d'onde moyenne). De manière générale, les situations quasi-forcées se révèlent être plus réalistes que les situations purement auto-organisées.

L'étude de modélisation numérique de TIESSEN et al. (2012) soulève l'importance de considérer la morphologie préexistante pour expliquer la formation des courants d'arrachement. En particulier, cette étude montre que la longueur d'onde des courants obtenus peut dépendre de la longueur d'onde présente dans le système morphologique préexistant, même si l'amplitude des perturbations bathymétriques est faible (indétectable avec les appareils de mesure).

Plusieurs études ont analysé l'effet d'un forçage de vague non-uniforme sur le développement des courants d'arrachement, en considérant un unique changement de climat (SMIT et al., 2005), une variation temporelle à l'échelle de régimes de vagues (1-10 jours, CASTELLE \& RUESSINK, 2011) ou de groupes de vagues (10-100 secondes, RENIERS et al., 2004). A la différence des autres études, RENIERS et al. (2004) prennent aussi en compte la variation spatiale des vagues, et montre que des 
cellules de circulations peuvent apparaitre si on moyenne le courant sur $15 \mathrm{mn}$. Ces cellules ont une influence sur la longueur $\mathrm{d}$ 'onde des systèmes rythmiques.

Le couplage morphologique introduit par CASTELLE et al. (2010) caractérise un forçage hydrodynamique provoqué par un système de barres rythmiques externes sur la barre interne. Ce mécanisme peut aussi être vu comme un mécanisme quasi-forcé puisque des courants d'arrachement se formant sur la barre interne peuvent aussi apparaitre sans forçage extérieur, même si le système externe a une forte influence sur la dynamique du système interne. Il faut ajouter que, même si dans leurs simulations le système externe a été créé artificiellement, on peut supposer qu'il aurait pu être généré préalablement par des processus d'auto-organisation durant des conditions de vagues plus énergétiques que les conditions étudiées.

\section{Conclusions}

Selon les dernières études de stabilité hydrodynamique, l'hypothèse de formation des courants d'arrachement bien établis (non épisodiques) la plus probable est l'hypothèse d'auto-organisation morphodynamique, pour les plages sableuses ouvertes. Ils seraient générés par des interactions entre le mouvement du fond, le transport sédimentaire et l'hydrodynamique.

Nous avons identifié les mécanismes quasi-forcés, où l'auto-organisation morphodynamique peut être influencée par une non-uniformité spatiale ou temporelle, soit dans les conditions initiales (bathymétrie initiale), soit dans le forçage (conditions de houle variables, influence de la morphologie extérieure). Il apparaît important de considérer ces non-uniformités pour simuler correctement la formation et le développement des courants d'arrachement.

Sous l'influence d'un forçage hydrodynamique extérieur (par un système de barres rythmiques sur la barre externe), lorsque la morphologie de la barre interne se réorganise pour former des structures morphologiques avec des courants d'arrachement associés, on parle de couplage morphologique, et les mécanismes sont quasi-forcés. Cependant il ne faut pas déprécier les régimes transitoires qui peuvent être observés avant que la barre interne se restructure. Ainsi, des courants d'arrachement transitoires peuvent se former, par des mécanismes entièrement forcés. Une étude spécifique est nécessaire, et les résultats obtenus lors de l'expérience en bassin présentée par MICHALLET et al. (2010) et par CASTELLE et al. (2010b) sont très prometteurs.

Enfin, même si les dernières études réalisées avec des modèles numériques 2DH montrent que les interactions entre le déferlement des vagues et les variations du niveau d'eau moyen empêchent la formation de courants d'arrachement, contestant ainsi l'hypothèse d'instabilités purement hydrodynamiques, l'exploration de cette hypothèse avec des modèles tridimensionnels (KUMAR et al., 2011) est un sujet ouvert. 


\section{XII ${ }^{\text {èmes }}$ Journées Nationales Génie Côtier - Génie Civil \\ Cherbourg, 12-14 juin 2012}

\section{Remerciements :}

Les travaux de R. Garnier ont été financés par le Ministère d'Économie et Compétitivité espagnol au sein du programme Juan de la Cierva. Les auteurs remercient L. Tarrade pour la relecture du manuscrit.

\section{Références bibliographiques}

BARCILON A.I., LAU J.P. (1973). A model for formation of transverse bars. J. Geophys. Res, Vol. 78, pp 2656-2664. doi:10.1029/JC078i015p02656

BLONDEAUX P. (2001). Mechanics of coastal forms. Annu. Rev. Fluid Mech., Vol. 33, pp 339-370. doi:10.1146/annurev.fluid.33.1.339

BOWEN A.J., INMAN D.L. (1969). Rip Currents. 2. Laboratory and Field Observations. J. Geophys. Res., Vol. 74, pp 5479-5490.

BOWEN A.J., INMAN D.L. (1971). EdgeWaves and Crescentic Bars. J. Geophys. Res., Vol. 76, pp 8662-8671. doi:10.1029/JC076i036p08662

CABALlERIA M., COCO G., FALQUÉS A., HUNTLEY D.A. (2002) Selforganization mechanisms for the formation of nearshore crescentic and transverse sand bars. J. Fluid Mech., Vol. 465, pp 379-410. doi:10.1017/S002211200200112X

CALVETE D., DODD N., FALQUÉS A., VAN LEEUWEN S.M. (2005). Morphological development of rip channel systems: Normal and near-normal wave incidence. J. Geophys. Res., 110, C10006. doi:10.1029/2004JC002803

CASTELLE B., BONNETON P., DUPUIS H., SÉNÉCHAL N. (2007). Double bar beach dynamics on the high-energy meso-macrotidal French Aquitanian Coast: A review. Mar. Geol., Vol. 245, pp 141-159. doi:10.1016/j.margeo.2007.06.001

CASTELlE B., RUESSINK B.G., BONNETON P., MARIEU V., BRUNEAU N., PRICE T.D. (2010). Coupling mechanisms in double sandbar systems. Part 2: Impact on alongshore variability of inner-bar rip channels. Earth Surf. Process. Landforms. doi:10.1002/esp.1949

CASTELlE B., MiCHALleT H., MARIEU V., LECKLER F., DUBARDIER B., LAMBERT A., BERNI C., BONNETON P., BARTHELEMY E., BOUCHETTE F. (2010b). Laboratory experiment on rip current circulations over a moveable bed: Drifter measurements. J. Geophys. Res., 115, C12008. doi:10.1029/2010JC006343

CASTELLE B., RUESSINK B.G. (2011). Modeling formation and subsequent nonlinear evolution of rip channels: time-varying versus time-invariant wave forcing. J. Geophys. Research. 116 (F04008). doi:10.1029/2011JF001997

CHRISTENSEN, E., DEIGAARD, R., FREDSOE, J. (1994). Sea bed stability on a long straight coast. In Proceedings Coastal Engineering 1994, ASCE, New York, Vol. 4, pp 1865-1879. 
COCO G., MURRAY A.B. (2007). Patterns in the sand: From forcing templates to self-organization. Geomorphology, Vol. 91, pp 271-290. doi:10.1016/j.geomorph.2007.04.023 DALRYMPLE R.A., LOZANO C.J. (1978). Wave-current interaction model for rip currents. J. Geophys. Res. Vol. 83, pp 6063-71. doi:10.1029/JC083iC12p06063

DALRYMPLE R.A., MACMAHAN J.H., RENIERS A.J.H.M, NELKO V. (2011). Rip Currents. Annu. Rev. Fluid Mech., Vol. 43, pp 551-81. doi:10.1146/annurev-fluid-122109-160733 DODD N., BLONDEAUX P., CALVETE D., DE SWART H.E., FALQUÉS A., HULSCHER S.J.M.H., RÓŻYŃSKI G., VITTORI G. (2003), Understanding coastal morphodynamics using stability methods. J. Coastal Res., Vol. 19(4), pp 849-865.

FALQUÉS A, MONTOTO A, VILA D. (1999). A note on hydrodynamic instabilities and horizontal circulation in the surf zone. J. Geophys. Res. Vol. 104, pp 20605-16. doi:10.1029/1999JC900120

FALQUÉS A, COCO G, HUNTLEY DA (2000) A mechanism for the generation of wave driven rhythmic patterns in the surf zone. J. Geophys. Res. Vol. 105(C10), pp 24071-24087. doi:10.1029/2000JC900100

FALQUÉS A., DODD N., GARNIER R., RIBAS F., MACHARDY F., SANCHO L.C., LARROUDÉ P., CALVETE D. (2008). Rhythmic surf-zone bars and morphodynamic self-organization. Coastal Eng., Vol. 55, pp 622-641. doi:10.1016/j.coastaleng.2007.11.012

GARNIER R., CALVETE D., FALQUÉS A., CABALLERIA M. (2006). Generation and non linear evolution of shore-oblique/transverse bars. J. Fluid Mech., Vol. 567: pp 327-360. doi:10.1017/S0022112006002126

GARNIER R., CALVETE D., FALQUÉS A., DODD N. (2008) Modelling the formation and the long-term behaviour of rip channel systems from the deformation of a longshore bar. J. Geophys. Res., 113, C07053. doi:10.1029/2007JC004632

GARNIER R., BONNETON P., FALQUÉS A., CALVETE D (2008b). Modélisation de la formation et de l'évolution non linéaire des barres en croissant de la cote aquitaine. La Houille Blanche, Vol. 3, pp 33-38. doi:10.1051/lhb:2008024

GARNIER R., DODD N., FALQUÉS A., CALVETE D. (2010) Mechanisms controlling crescentic bar amplitude. J. Geophys. Res., 115, F02007. doi:10.1029/2009JF001407

GUZA R.T., INMAN D.L (1975). Edge waves and beach cusps. J. of Geophys. Res., Vol. 80, pp 2997-3012. doi:10.1029/JC080i021p02997

HASAN H., DODD N., GARNIER R. (2009). Stabilizing effect of random waves on rip currents. J. Geophys. Res. 114:C07010. doi:10.1029/2008JC005031

HINO M. (1974). Theory on formation of rip-current and cuspidal coast. In Proceedings Coastal Engineering 1974, ASCE, New York, pp 901-919. 


\section{XII ${ }^{\text {èmes }}$ Journées Nationales Génie Côtier - Génie Civil \\ Cherbourg, 12-14 juin 2012}

KUMAR N., VOULGARIS G., WARNER J.C. (2011). Implementation and modification of a three-dimensional radiation stress formulation for surf zone and ripcurrent applications. Coastal Engineering, Vol. 58, pp 1097-1117. doi:10.1016/j.coastaleng.2011.06.009

LAFON V., DUPUIS H., HOWA H., FROIDEFOND J.M. (2002). Determining ridge and runnel longshore migration rate using spot imagery. Oceanol. Acta, Vol. 25, pp 149-158. doi:10.1016/S0399-1784(02)01190-8

MACMAHAN J., THORNTON E.B., STANTON,T.P., RENIERS A.J.H.M. (2005). RIPEX: Observations of a rip current system. Mar. Geol., Vol. 218, pp 113- 134. doi:10.1016/j.margeo.2005.03.019

MACMAHAN J.H., THORNTON E.B., RENIERS A.J.H.M (2006). Rip current review. Coast. Eng. Vol. 53, pp 191- 208. doi:10.1016/j.coastaleng.2005.10.009

Michallet H., CASTElle B., BOUChETTE F., LAMBERT A., BERNI C., BARTHELEMY E., BONNETON P., SOUS D. (2010). Modélisation de la morphodynamique d'une plage barrée tridimensionnelle. XI Journées National Génie Côtier-Génie Civil, pp 379-386. doi:10.5150/jngcgc.2010.045-M

RENIERS A.J.H.M., ROELVINK J.A., THORNTON E.B. (2004), Morphodynamic modeling of an embayed beach under wave group forcing, J. Geophys. Res., 109, C01030. doi:10.1029/2002JC001586

RIBAS F., FALQUÉS A., MONTOTO A. (2003). Nearshore oblique sand bars. J. Geophys. Res. 108, 3119. doi:10.1029/2001JC000985

SHORT A.D. (1999). Handbook of Beach and Shoreface Morphodynamics. Wiley, Chichester, U.K.

SMIT M.W.J., RENIERS A.J.H.M., STIVE M.J.F. (2005). Nearshore bar response to time varying conditions. In Coastal Dynamics 2005, ASCE. doi:10.1061/40855(214)74

SONU C.J. (1968). Collective movement of sediment in littoral environment. In Proceedings Coastal Engineering 1968, ASCE, New York, pp 373-400.

SONU C.J. (1972). Comment on Paper by A. J. Bowen and D. L. Inman, 'Edge Wave and Crescentic Bars'. J. Geophys. Res. Vol. 77, pp 6629-6631. doi:10.1029/JC077i033p06629 THIEBOT J., IDIER D., GARNIER R., FALQUÉS A., RUESSINK B.G. (2012) The influence of wave direction on the morphological response of a double sandbar system. Continental Shelf Research, Vol. 32, pp 71-85. doi:10.1016/j.csr.2011.10.014

THORNTON E.B., GUZA R.T. (1983). Transformation of wave height distribution, J. Geophys. Res. Vol. 88, pp 5925-5938. doi:10.1029/JC088iC10p05925

TIESSEN M.C.H., DODD N., GARNIER R. (2011). Development of crescentic bars for a periodically perturbed initial bathymetry. J. Geophys. Res., 116, F04016. doi:10.1029/2011JF002069 
Thème 1 - Hydrodynamique côtière

VAN ENCKEVORT I.M.J., RUESSINK B.G., COCO G., SUZUKI K., TURNER I.L., PLANT N.L., HOLMAN R.A. (2004). Observations of nearshore crescentic sandbars. J. Geophys. Res., 109, C06028. doi:10.1029/2003JC002214

YU J. (2006). On the instability leading to rip currents due to wave-current interaction.

J. Fluid Mech, Vol. 549, pp 403-428. doi:10.1017/S0022112005008335 